\title{
Annexin A10 Expression Is Associated With Poor Prognosis in Small Bowel Adenocarcinoma
}

\author{
AKIRA ISHIKAWA ${ }^{1}$, KAZUYA KURAOKA ${ }^{1,2 \#}$, JUNICHI ZAITSU ${ }^{2}$, AKIHISA SAITO $^{2}$, TOSHIO KUWAI ${ }^{3}$, \\ YOSUKE SHIMIZU ${ }^{4}$, TAKESHI SUDO ${ }^{4}$, HIROTAKA TASHIRO $^{4}$, KIYOMI TANIYAMA $^{5}$ and WATARU YASUI ${ }^{6}$ \\ ${ }^{1}$ Institute for Clinical Laboratory, National Hospital Organization, \\ Kure Medical Center and Chugoku Cancer Center, Kure, Japan; \\ ${ }^{2}$ Department of Diagnostic Pathology, National Hospital Organization, \\ Kure Medical Center and Chugoku Cancer Center, Kure, Japan; \\ ${ }^{3}$ Department of Gastroenterology, National Hospital Organization, \\ Kure Medical Center and Chugoku Cancer Center, Kure, Japan; \\ ${ }^{4}$ Department of Surgery, National Hospital Organization, \\ Kure Medical Center and Chugoku Cancer Center, Kure, Japan; \\ ${ }^{5}$ Honorary President, National Hospital Organization, \\ Kure Medical Center and Chugoku Cancer Center, Kure, Japan; \\ ${ }^{6}$ Department of Molecular Pathology, Graduate School of Biomedical and Health Sciences, \\ Hiroshima University, Hiroshima, Japan
}

\begin{abstract}
Background/Aim: Small bowel adenocarcinoma $(S B A)$ is a relatively rare malignant epithelial neoplasm. Thus, little is known about prognostic biomarkers of SBA. Annexin A10 (ANXA10) is a member of the annexin family. The significance of ANXA10 expression in SBA is unclear. This is the first study to examine the expression of ANXA10 in SBA. Materials and Methods: We immunohistochemically evaluated ANXA10 expression of SBA and studied the relationship between ANXA10 expression and clinicopathological factors. Results: ANXA10 expression was detected in 17 (56.7\%) of 30 SBA cases and was significantly associated with poor overall survival. Univariate predictors for poor prognosis were tumour size $(p=0.017)$ and ANXA10 expression $(p=0.026)$. In multivariate analysis, ANXA10 expression $(p=0.038)$ and tumour size $(p=0.024)$ were found to be independent predictors of poor prognosis. Conclusion: ANXA10 could be a new prognostic biomarker for SBA.
\end{abstract}

This article is freely accessible online.

Correspondence to: Kazuya Kuraoka, Department of Diagnostic Pathology, National Hospital Organization, Kure Medical Center and Chugoku Cancer Center,3-1 Aoyama, Kure 737-0023, Japan. Tel: +81 823223111, Fax: +81 823210478, e-mail: kuraoka.kazuya.vk@mail.hosp.go.jp

Key Words: Annexin A10, small bowel adenocarcinoma, nonampullary adenocarcinoma, prognostic biomarker.
Small bowel adenocarcinoma (SBA) is a relatively rare malignant epithelial neoplasm. The small intestine, consisting of the duodenum, jejunum, and ileum, represents $75 \%$ of the length of the gastrointestinal tract, extending from the pylorus to the ileocecal valve. Less than $5 \%$ of the total annual gastrointestinal cancer incidence occurs in the small intestine (1). The frequency of small bowel cancer is 1.2-2.3 cases per 100000 person-years (2). The most common subtype is SBA, which accounts for 30-40\% of small bowel cancers (3). Many aspects are common between SBA and colorectal cancers (CRCs), including geographical correlation in incidence rates (4). Several genetic factors similar to those in CRCs, such as TP53 (5) and CTNNB1 (encoding $\beta$-catenin) (6), have been reported. Due to these similarities, the management of SBA has been based on that of CRCs (7). However, in contrast to CRCs, there is considerable room for improvement in the management of SBA. In a recent metanalysis of unresectable or metastatic SBA chemotherapy, the objective response rate and the disease control rate were $18-50 \%$ and $29-87 \%$, respectively (7). Therefore, a prognostic biomarker of SBA is required.

Annexin A10 (ANXA10) is a member of the annexin family and a calcium- and phospholipid-binding protein. Annexin family proteins are characterized by their signature feature, the annexin repeat (8), and have diverse and crucial functions in cellular and physiological processes $(9,10)$. In normal organs, the expression of ANXA10 is detected in the stomach, bladder, kidney, urinary bladder, and duodenum. In the duodenum, ANXA10 expression is limited to the nuclei of Brunner gland cells but is not observed in the mucosal epithelia (11). 
Several carcinomas have been reported to express ANXA10, including carcinomas of the liver (12), gastrointestine (11), and oesophagus (13). We have previously reported that ANXA10 is associated with the gastric phenotype of gastric cancer through the regulation of pancreatic duodenal homeobox-1 (PDX1) (14) and that ANXA10 is associated with early-stage gastric cancer (15). Previous studies have suggested that some SBA cases have been known to express gastric mucin (MUC5AC and MUC6) and have the gastric phenotype (16). However, the significance of ANXA10 expression in normal small intestine and SBA has not been investigated. This is the first study to investigate the expression of ANXA10 in SBA.

In the current study, we aimed to investigate the expression and prognostic value of ANXA10 in SBA by examining its expression by immunohistochemistry (IHC) in surgically or endoscopically resected tissue samples from 30 cases Furthermore, we examined the correlations between the expression of ANXA10 protein and clinicopathological features. Lastly, we investigated the relationship between ANXA10 expression and prognosis of SBA cases.

\section{Materials and Methods}

Tissue samples. In this retrospective study, 30 primary SBA tissue samples were gathered from SBA patients, who underwent surgical or endoscopic resection between January 2002 and December 2019 at the Kure Medical Center and Chugoku Cancer Center (Hiroshima, Japan). Patients were observed by their physicians until their death or the date of the last documented contact. For IHC, the collected tissue samples were archival formalin-fixed and paraffinembedded. One representative tumour block from each patient was evaluated by IHC. The tumour stage was determined according to the TNM classification system. Histological classifications were determined based on the guidelines of the Japanese Society for the Colon and Rectum due to a lack of SBA-specific guidelines. The Ethical Committee for Human Genome Research of Kure Medical Center and Chugoku Cancer Center (2019-91) approved the present study and all patient samples were obtained with consent.

Immunohistochemistry. IHC staining was performed based on previously described methods with some modifications $(14,15)$. For IHC evaluation, paraffin-embedded tissue samples from 30 representative blocks were cut into $4 \mu \mathrm{m}$-thick sections, deparaffinized, and rehydrated. IHC staining was performed using a Ventana Benchmark ULTRA auto-stainer (Ventana Medical Systems, Tucson, AZ, USA) and the labelled streptavidin-biotin peroxidase method, and signals were visualized with 3,3'diaminobenzidine. For antigen retrieval mild and standard cell conditioning 1 buffer was used. Sections were incubated with a rabbit monoclonal anti-ANXA10 antibody (1:500, Novus Biologicals, Centennial, CO, USA, NBP1-90156) for $32 \mathrm{~min}$. Endogenous peroxidase activity was not blocked. ANXA10 expression was scored in all tumours as positive or negative. The specificity of the antibody has been previously confirmed (5). The endogenous positive control was the Brunner's gland in the same section, while the negative control was fibroblasts. When $>10 \%$ of

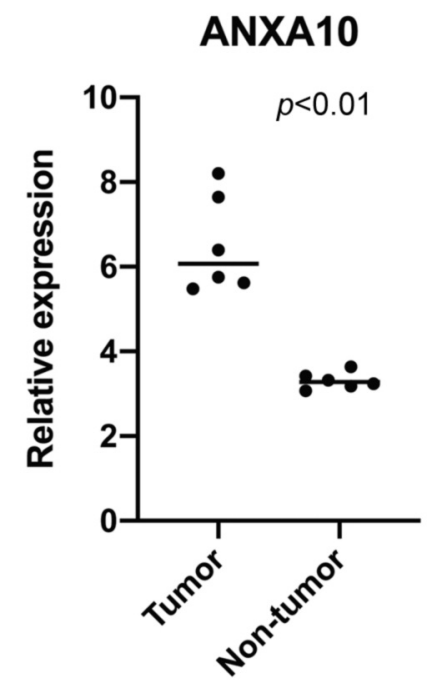

Figure 1. Annexin A10 (ANXA10) expression was detected in mouse small intestinal tumours. GEO2R analysis of six non-neoplastic and six neoplastic tissue samples from GSE111594.

tumour cells were stained, the immunostaining was regarded positive for ANXA10. Based on these rules, two surgical pathologists (A.I. and K.K.), with no knowledge of the clinical and pathologic parameters or patient outcomes, independently reviewed the immunoreactivity of each specimen.

Microarray dataset analysis. We used the GSE dataset (GSE111594) comprising six mouse intestinal tumour and six crypt cell samples, which was downloaded from the GEO database (17) and analysed using GEO2R.

Statistical analysis. A $p<0.05$ was considered to indicate a statistically significant difference. We used Fisher's exact test to examine correlations between clinicopathological parameters and ANXA10 expression. To test the statistical differences between survival curves the log-rank test was used. Univariate and multivariate Cox regressions were tested for the associations between clinical covariates and overall survival. The hazard ratio and $95 \%$ confidence intervals were calculated from Cox proportional hazard models.

\section{Results}

ANXA10 expression was identified in mouse intestinal tumours. First, to examine the expression of ANXA10 in small intestinal tumours, we analysed the levels of ANXA10 expression in six crypt cells and six tumour cells in the Gene Expression Omnibus (GEO) database with accession no. GSE111594 using GEO2R. The ANXA10 levels were significantly higher in tumour cells than crypt cells (Figure 1). This result supported the hypothesis that ANXA10 expression, which is not expressed in normal small intestine, could be expressed in small intestinal tumours. 
Table I. Patient characteristics.

\begin{tabular}{|c|c|}
\hline All & Total 30 cases $(\%)$ \\
\hline \multicolumn{2}{|l|}{ Age (years) } \\
\hline Mean (range) & $65.4(61-70)$ \\
\hline \multicolumn{2}{|l|}{ Gender } \\
\hline Male & $17(56.7)$ \\
\hline Female & 13 \\
\hline \multicolumn{2}{|l|}{ Size $(\mathrm{cm})$} \\
\hline Mean (range) & $3.7(2.5-4.9)$ \\
\hline \multicolumn{2}{|l|}{ Site } \\
\hline Duodenum & $21(70)$ \\
\hline Ileum/Jejunum & $9(30)$ \\
\hline \multicolumn{2}{|l|}{ pT } \\
\hline $\mathrm{pT} 1 / 2$ & $12(40)$ \\
\hline $\mathrm{pT} 3 / 4$ & 18 \\
\hline \multicolumn{2}{|l|}{$\mathrm{pN}$} \\
\hline pNO & 21 \\
\hline $\mathrm{pN} 1 / 2 / 3$ & $9(30)$ \\
\hline \multicolumn{2}{|l|}{ Histological type } \\
\hline Well differentiated tubular & $16(53)$ \\
\hline Moderately differentiated tubular & $6(20)$ \\
\hline Papillary (pap) & $3(10)$ \\
\hline Mucinous (muc) & $2(6)$ \\
\hline \multicolumn{2}{|l|}{ Lymphatic invasion (Ly) } \\
\hline Ly0 & 19 \\
\hline Ly1 & $11(36.7)$ \\
\hline \multicolumn{2}{|l|}{ Vascular invasion $(\mathrm{V})$} \\
\hline V0 & 24 \\
\hline V1 & $6(20)$ \\
\hline \multicolumn{2}{|l|}{ ANXA10 expression } \\
\hline Positive & $17(56.7)$ \\
\hline Negative & 13 \\
\hline
\end{tabular}

Clinicopathological features of recruited patients. To examine the clinicopathological features of SBA, we recruited 30 patients diagnosed with SBA in our institute. The clinicopathological features are summarized in Table I. The mean age was 65.4 years (range $=61-70$ years), and $17(56.7 \%)$ patients were male. The mean of tumour size was $3.7 \mathrm{~cm}$ (range $=2.5-4.9 \mathrm{~cm})$. Regarding the primary tumour site, 21 $(70 \%)$ were in the duodenum, and in the other $9(30 \%)$ cases was in the ileum or jejunum. In terms of the pathological features and TNM staging, 12 (40\%) cases were diagnosed with pT1 or pT2, whereas another 18 cases were pT3 or pT4. Nine $(30 \%)$ cases had lymph node metastasis. Regarding the histological subtype, $16(53 \%)$ cases were well differentiated, $6(20 \%)$ cases were moderately differentiated, $3(10 \%)$ cases were papillary, and $2(6 \%)$ cases were mucinous adenocarcinoma. Lymphatic invasion and vascular invasion were observed in $11(36.7 \%)$ and $6(20 \%)$ cases, respectively. ANXA10 expression was detected in 17 (56.7\%) cases.

ANXA10 expression in the small intestine and SBA. To investigate the clinicopathological significance of ANXA10
Table II. The relationship between ANXA10 expression and clinicopathological features in patients with small bowel adenocarcinoma.

\begin{tabular}{|c|c|c|c|}
\hline & \multicolumn{2}{|c|}{ Annexin A10 expression } & \multirow[b]{2}{*}{$p$-Value } \\
\hline & Positive $(\%)$ & Negative & \\
\hline Age (years) & & & 1.000 \\
\hline$<65$ & $10(58)$ & 8 & \\
\hline$\geq 65$ & $7(58)$ & 5 & \\
\hline Gender & & & 1.000 \\
\hline Male & $9(60)$ & 6 & \\
\hline Female & $8(53)$ & 7 & \\
\hline Size $(\mathrm{cm})$ & & & 0.462 \\
\hline$<3.7$ & $7(47)$ & 8 & \\
\hline$\geq 3.7$ & $10(67)$ & 5 & \\
\hline pT & & & 1.000 \\
\hline $\mathrm{pT} 1 / 2$ & $7(58)$ & 5 & \\
\hline $\mathrm{pT} 3 / 4$ & $10(58)$ & 8 & \\
\hline $\mathrm{pN}$ & & & 1.000 \\
\hline pNO & $12(71)$ & 9 & \\
\hline $\mathrm{pN} 1 / 2 / 3$ & $5(56)$ & 4 & \\
\hline $\mathrm{V}$ & & & 0.672 \\
\hline V0 & $13(54)$ & 11 & \\
\hline V1 & $4(67)$ & 2 & \\
\hline Ly & & & 0.462 \\
\hline Ly0 & $10(53)$ & 9 & \\
\hline Ly1 & $7(64)$ & 4 & \\
\hline
\end{tabular}

expression, we performed IHC staining for ANXA10 in 30 SBA tissue samples, including non-neoplastic tissue. In nonneoplastic duodenal tissue, ANXA10 expression was observed in Brunner glands, as has been reported (11), but not in absorptive cells or goblet cells (Figure 2A). ANXA10 expression in Brunner glands was heterogeneous and was found in the nuclei of the gland cells (Figure 2B). No ANXA10 expression was observed in either the jejunum or ileum (Figure 2C and D). In SBA tissue, ANXA10 expression was detected in the nuclei and cytoplasm of tumour cells (Figure 2E and F). We defined immunostaining as positive if $10 \%$ of the SBA cancer cell nuclei showed visible signals, according to previous studies $(14,15)$. A total of $17(56.7 \%)$ SBA samples showed ANXA10 expression. Several SBA samples demonstrated ANXA10 staining heterogeneity, and heterogeneous ANXA10 expression with no trend was observed.

Correlation between ANXA10 expression and clinicopathological features in SBA. To determine the relationship between ANXA10 staining and clinicopathologic features (Table II) we performed that Fisher's exact test, which demonstrated no significant difference between ANXA10 expression and any factors, including age $(p=1.000)$, sex $(p=1.000)$, size $(p=0.462), \mathrm{pT}(p=1.000), \mathrm{pN}(p=1.000), \mathrm{V}$ $(p=0.672)$, or Ly $(p=0.462)$. 

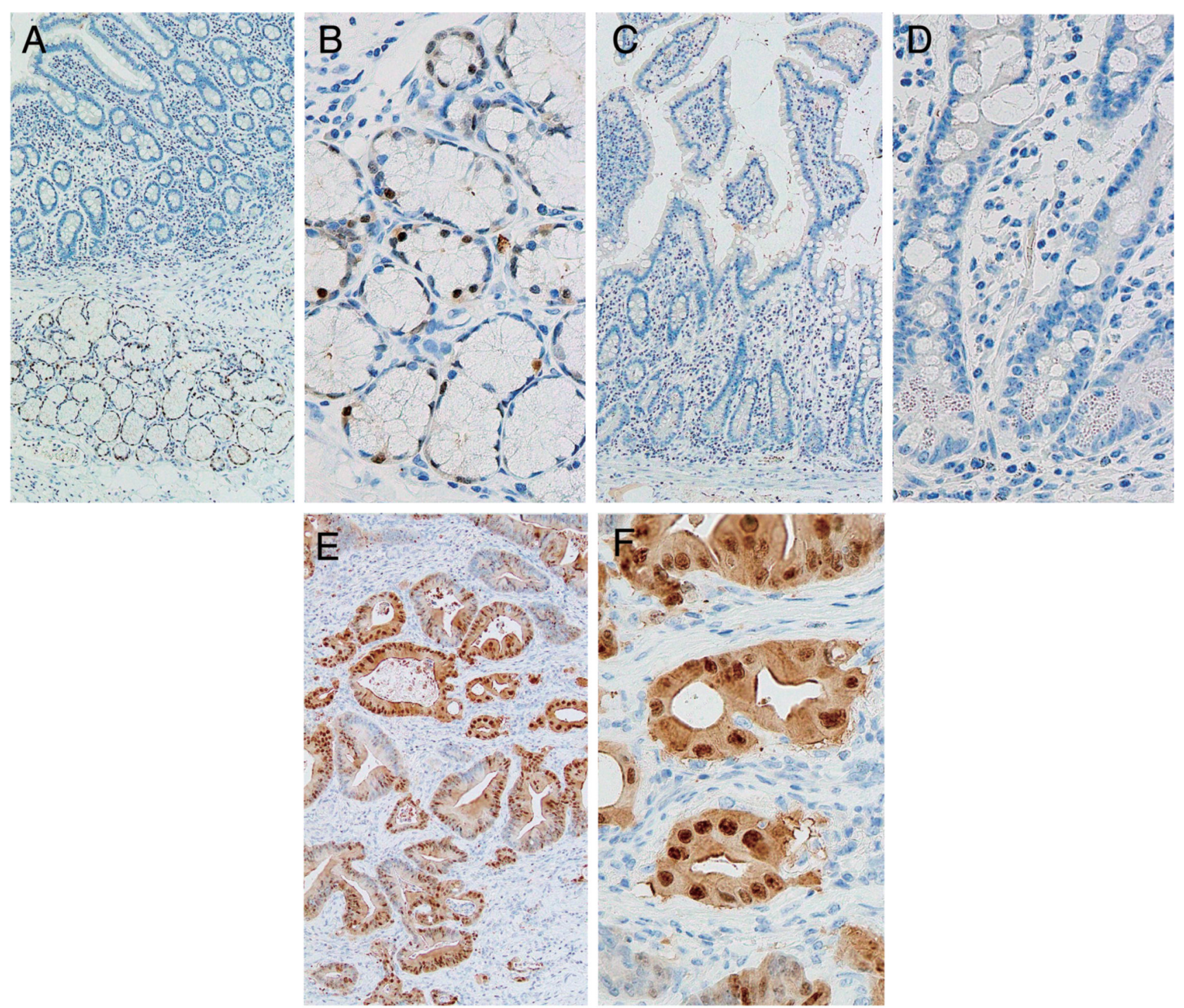

Figure 2. Representative annexin A10 (ANXA10) images in non-neoplastic small intestinal mucosa and small bowel adenocarcinoma (SBA). (A, B) Immunohistochemical staining for ANXA10 in non-neoplastic duodenal mucosa. Original magnification: (A) 100x and (B) 400x. (C, D) Immunohistochemical staining for ANXA10 in non-neoplastic jejunal mucosa. Original magnification: (C) 100x and (D) 400x. (E, F) Immunohistochemical staining for ANXA10 in SBA. Original magnification: $(E)$ 100x and $(F) 400 \times$.

Correlation between ANXA10 expression and survival of SBA patients. The 5-year overall survival (OS) rate was $36.7 \%$ for ANXA10-positive cases and $82.5 \%$ for ANXA10negative cases. ANXA10-positive SBA cases had significantly poorer survival probability than ANXA10negative cases ( $p=0.030$; Figure $3 \mathrm{~A}$ ). The 5 -year disease-free survival (DFS) rate of ANXA10-positive cases was 34.0\%, and that of ANXA10-negative cases was $75.5 \%$. A marginally significant difference was observed in the 5-year DFS rate $(p=0.070$; Figure 3B). Additionally, the same examination as ANXA10 was conducted for size. The cases with tumours larger than the median $3.7 \mathrm{~cm}$ were categorized as large, and those smaller than $3.7 \mathrm{~cm}$ were categorized as small. The 5-year OS rate was $31.5 \%$ for large tumours and $86.2 \%$ for small tumours. Large tumour SBA cases had significantly poorer survival probability than those with small tumours $(p=0.019$; Figure $3 \mathrm{C})$. The 5 -year DFS rates were $28.6 \%$ and $79.0 \%$ for large and small tumors, respectively. A significant difference was observed in the 5year DFS rate $(p<0.01$; Figure 3D).

The results of the univariate and multivariate Cox proportional hazard analyses to assess ANXA10 expression as a prognostic indicator are presented in Table III. In univariate analysis, tumour size $(\mathrm{HR}=5.350 ; 95 \% \mathrm{CI}=1.324-$ 
A

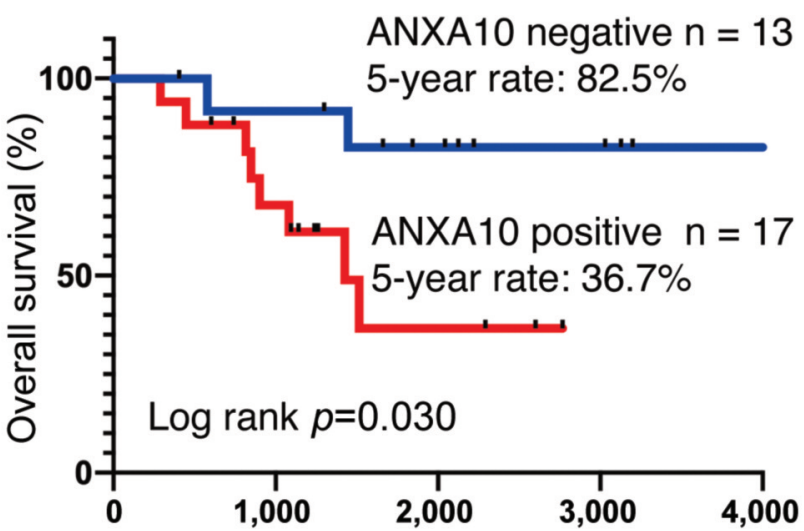

C

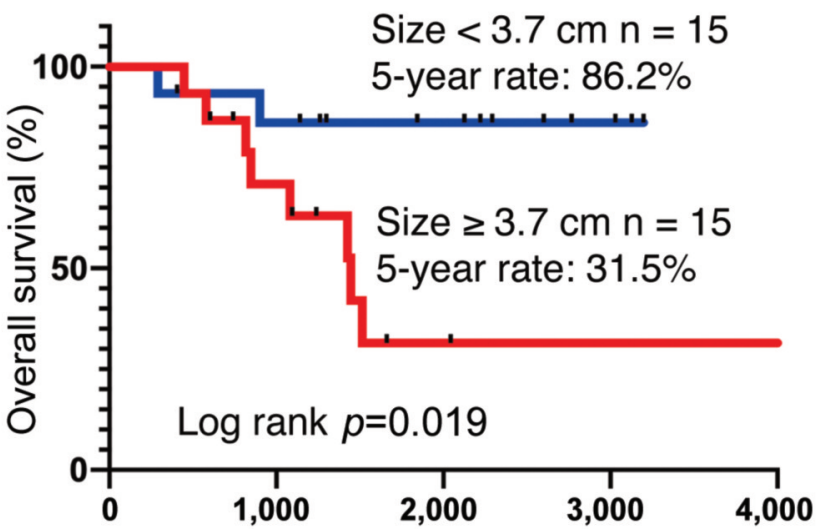

B

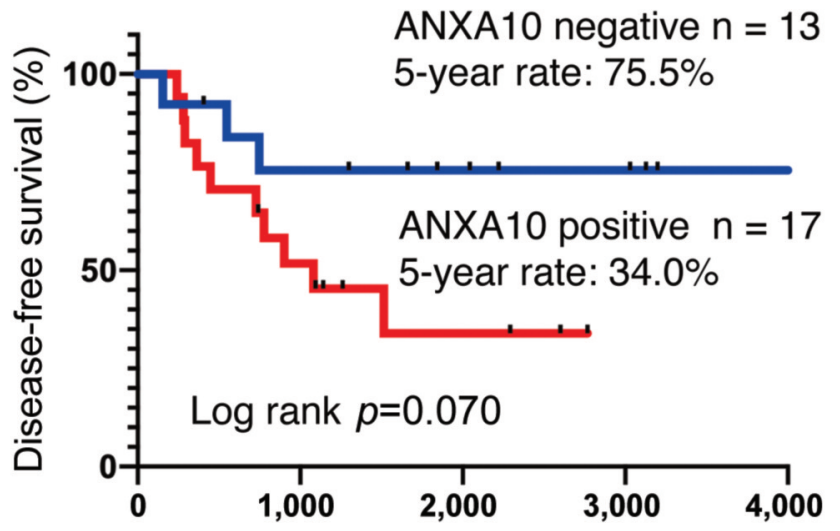

D

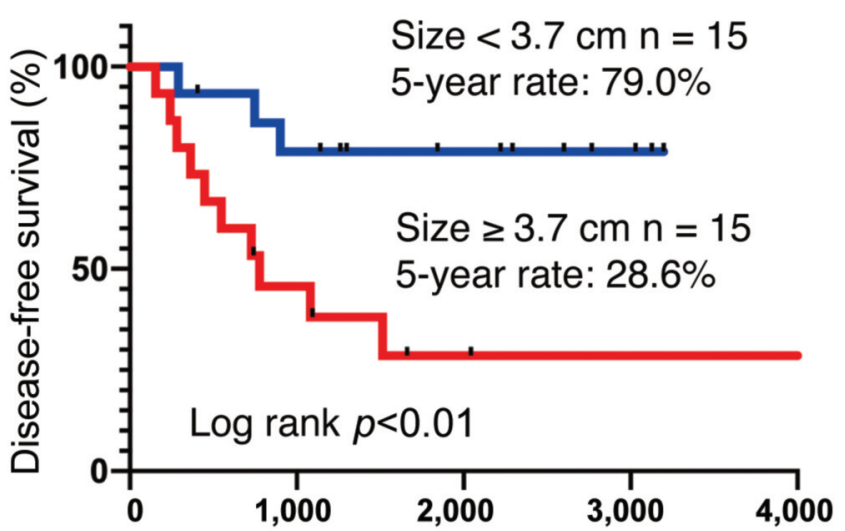

Figure 3. Kaplan-Meier curves of 30 small bowel adenocarcinoma (SBA) cases. Annexin A10 (ANXA10)-positive and -negative SBA cases of (A) Overall survival (OS) and (B) disease-free survival (DFS). (C) OS and (D) DFS of large tumour and small tumour SBA cases.

$35.69 ; p=0.017)$ and ANXA10 expression $(\mathrm{HR}=4.926$; 95\% CI=1.194-33.28; $p=0.026$ ) were associated with poor survival. In multivariate analysis, tumour size $(\mathrm{HR}=5.000$; 95\% CI $=1.215-33.72 ; p=0.024)$ and ANXA10 expression $(\mathrm{HR}=4.634 ; 95 \% \mathrm{CI}=1.085-32.00 ; p=0.038)$ were found to be independent predictors of survival in SBA patients.

\section{Discussion}

In the current study, we analysed the significance and expression of ANXA10 in SBA. To our knowledge, this is the first study that demonstrates the localization of ANXA10 in the small intestine and examines its significance in SBA.

A previous study has reported that, in non-neoplastic tissue, duodenal ANXA10 expression was found in the Brunner glands (11). Consistently, the expression of ANXA10 was limited to the nuclei of Brunner gland cells in the small intestine. There has been no report of ANXA10 expression in SBA. Organs close to the small intestine have been reported to be positive in $46-74.6 \%$ of gastric cancers $(11,14,18,19)$ and $11-17 \%$ of CRCs $(20,21)$. In particular, ANXA10 expression was observed more frequently (up to $87.5 \%$ ) in CRCs of the gastric phenotype than in conventional CRCs (20-22). Therefore, some SBAs with the gastric phenotype may have different carcinogenic pathways than other CRCs.

In the present study, univariate and multivariate analyses showed that large tumour size and ANXA10 expression correlated with OS and acted as an independent prognostic classifier of patients with SBA. In view of tumour size, a size $\geq 5 \mathrm{~cm}$ has been suggested to influence survival time (23). However, the most interesting observation was the significance of ANXA10 expression in SBA. Previous reports have shown that ANXA10 is an independent prognostic factor in several carcinomas: hepatocellular carcinoma (12), cholangiocarcinoma (24), ovarian cancer (25), thyroid cancer (26), and early gastric cancers (15). These results suggest that ANXA10 immunostaining is a clinically significant modality to predict survival of SBA patients. 
Table III. Univariate and multivariate Cox regression analyses of ANXA10 expression and survival of small bowel adenocarcinoma patients.

\begin{tabular}{|c|c|c|c|c|}
\hline \multirow[b]{2}{*}{ Features } & \multicolumn{2}{|c|}{ Univariate analysis } & \multicolumn{2}{|c|}{ Multivariate analysis } \\
\hline & $\mathrm{HR}(95 \% \mathrm{CI})$ & $p$-Value & HR $(95 \% \mathrm{CI})$ & $p$-Value \\
\hline \multicolumn{5}{|l|}{ Age } \\
\hline$<65$ & 1 (ref.) & 0.571 & & \\
\hline$\geq 65$ & $1.432(0.400-5.166)$ & & & \\
\hline Size & & 0.017 & & 0.024 \\
\hline$<3.7 \mathrm{~cm}$ & 1 (ref.) & \multirow{2}{*}{\multicolumn{3}{|c|}{$\begin{array}{c}1 \text { (ref.) } \\
5.000(1.215-33.72)\end{array}$}} \\
\hline$\geq 3.7 \mathrm{~cm}$ & $5.350(1.324-35.69)$ & & & \\
\hline Gender & & \multicolumn{3}{|c|}{$0(1.215-33.12)$} \\
\hline Female & 1 (ref.) & & & \\
\hline Male & $2.414(0.067-11.26)$ & \multirow{2}{*}{\multicolumn{3}{|c|}{0122}} \\
\hline pT & & & & \\
\hline $\mathrm{pT} 1 / 2$ & 1 (ref.) & & & \\
\hline $\mathrm{pT} 3 / 4$ & $2.978(0.740-19.83)$ & \multirow{2}{*}{\multicolumn{3}{|c|}{0.186}} \\
\hline $\mathrm{pN}$ & & & & \\
\hline pNO & 1 (ref.) & & & \\
\hline $\mathrm{pN} 1 / 2 / 3$ & $2.343(0.650-8.453)$ & \multirow{2}{*}{\multicolumn{3}{|c|}{0.064}} \\
\hline $\mathrm{V}$ & & & & \\
\hline V0 & 1 (ref.) & & & \\
\hline V1 & $3.707(0.918-13.56)$ & & & \\
\hline \multicolumn{5}{|c|}{ ) } \\
\hline Ly0 & 1 (ref.) & \multirow{2}{*}{\multicolumn{3}{|c|}{0.093}} \\
\hline Ly1 & $2.942(0.831-11.62)$ & & & \\
\hline \multicolumn{5}{|c|}{$\begin{array}{l}\text { ANXA10 } \\
\text { expression }\end{array}$} \\
\hline Negative & 1 (ref.) & 0.026 & 1 (ref.) & 0.038 \\
\hline Positive & $4.926(1.194-33.28)$ & \multicolumn{3}{|c|}{$4.634(1.085-32.00)$} \\
\hline
\end{tabular}

Regarding the association between ANXA10 expression and clinicopathological features, no correlation was detected between ANXA10 expression and other clinicopathological features despite survival significance. In previous studies, ANXA10 expression has been associated with tumour stage, lymph node metastasis, and distant metastasis in gastric cancers $(14,19)$ and with gender, tumour stage, tumour location, tumour differentiation, and tumour histology in CRCs $(21,22)$. In SBA, similar relationships are hypothesized with other gastrointestinal cancers, but further study with larger numbers of cases is needed to investigate their effects on tumour progression.

The most significant limitations in the current study are that this is a single institute study and the number of cases is small. However, given the fact that SBA is a very rare disease (1), our analysis provides new evidence for ANXA10 involvement in the pathogenesis of SBA. Another limitation of this study is that the specific role of ANXA10 in SBA was not examined. The function of ANXA10 has been reported in several organs (13, 14, 20, 21). In CRCs, ANXA10 expression has been correlated with microsatellite instability (21) and involves BRAF mutation and $\mathrm{CpG}$ island methylation (20). In oesophageal cancer, ANXA10 promotes cell proliferation through phosphorylation of Akt and Erk1/2 (13). In fact, we have previously reported that ANXA10 is involved in the gastric phenotype via PDX1 in advanced gastric cancer (14). As with gastric cancer, there are some SBAs that have gastric phenotype (27), and the role of ANXA10 may be different in each phenotype. Identification of ANXA10 downstream signalling will further improve the understanding of the basic biology of ANXA10.

In conclusion, we demonstrated the clinical significance of ANXA10 in SBA patients. ANXA10- positive patients have significantly poor prognosis, and the expression status of ANXA10 is an independent prognostic marker. ANXA10 immunostaining could be a new prognostic indicator for SBA patients.

\section{Conflicts of Interest}

The Authors state that there are no conflicts of interest in relation to this study.

\section{Authors' Contributions}

AI designed the study. TK, YS, HT, and TS collected and analysed the patient clinical data. AI, KK, JZ, AS, and KT performed the experiments and collected and analysed the data. AI, KK, TK, YS, TS, HT, KT, and WY interpreted and analysed the results. AI, KK, and WY drafted and edited the manuscript. All Authors read and approved the manuscript and agree to be accountable for all aspects of the research in ensuring that the accuracy or integrity of any part of the work are appropriately investigated and resolved.

\section{Acknowledgements}

The Authors would like to thank the patients for allowing us to report their clinical information and data, and to express their sincere gratitude to Yasumura N, Kan A, Fujisawa H, Iwahiro K, and Kimura $\mathrm{F}$ for their support and excellent technical assistance.

\section{References}

1 Schottenfeld D, Beebe-Dimmer JL and Vigneau FD: The epidemiology and pathogenesis of neoplasia in the small intestine. Ann Epidemiol 19(1): 58-69, 2009. PMID: 19064190. DOI: 10.1016/j.annepidem.2008.10.004

2 Bilimoria KY, Bentrem DJ, Wayne JD, Ko CY, Bennett CL and Talamonti MS: Small bowel cancer in the United States: changes in epidemiology, treatment, and survival over the last 20 years. Ann Surg 249(1): 63-71, 2009. PMID: 19106677. DOI: 10.1097/ SLA.0b013e31818e 4641

3 Aparicio T, Zaanan A, Mary F, Afchain P, Manfredi S and Evans TR: Small Bowel Adenocarcinoma. Gastroenterol Clin North Am 45(3): 447-57, 2016. PMID: 27546842. DOI: 10.1016/j.gtc. 2016.04.004

4 Haselkorn T, Whittemore AS and Lilienfeld DE: Incidence of small bowel cancer in the United States and worldwide: geographic, temporal, and racial differences. Cancer Causes 
Control 16(7): 781-787, 2005. PMID: 16132788. DOI: 10.1007/ s10552-005-3635-6

5 Wheeler JM, Warren BF, Mortensen NJ, Kim HC, Biddolph SC, Elia G, Beck NE, Williams GT, Shepherd NA, Bateman AC and Bodmer WF: An insight into the genetic pathway of adenocarcinoma of the small intestine. Gut 50(2): 218-223, 2002. PMID: 11788563. DOI: 10.1136/gut.50.2.218

6 Bläker H, Helmchen B, Bönisch A, Aulmann S, Penzel R, Otto $\mathrm{HF}$ and Rieker RJ: Mutational activation of the RAS-RAF-MAPK and the Wnt pathway in small intestinal adenocarcinomas. Scand J Gastroenterol 39(8): 748-753, 2004. PMID: 15513360. DOI: $10.1080 / 00365520410005847$

7 Nishikawa Y, Hoshino N, Horimatsu T, Funakoshi T, Hida K, Sakai Y, Muto M and Nakayama T: Chemotherapy for patients with unresectable or metastatic small bowel adenocarcinoma: a systematic review. Int J Clin Oncol 25(8): 1441-1449, 2020. PMID: 32448950. DOI: 10.1007/s 10147-020-01703-Z

8 Schloer S, Pajonczyk D and Rescher U: Annexins in translational research: Hidden treasures to be found. Int J Mol Sci 19(6): 1781, 2018. PMID: 29914106. DOI: 10.3390/ ijms 19061781

9 Lizarbe MA, Barrasa JI, Olmo N, Gavilanes F and Turnay J: Annexin-phospholipid interactions. Functional implications. Int J Mol Sci 14(2): 2652-2683, 2013. PMID: 23358253. DOI: $10.3390 / \mathrm{ijms} 14022652$

10 Gerke V, Creutz CE and Moss SE: Annexins: linking Ca2+ signalling to membrane dynamics. Nat Rev Mol Cell Biol 6(6): 449-461, 2005. PMID: 15928709. DOI: 10.1038/nrm1661

$11 \mathrm{Lu} \mathrm{SH}$, Yuan RH, Chen YL, Hsu HC and Jeng YM: Annexin A10 is an immunohistochemical marker for adenocarcinoma of the upper gastrointestinal tract and pancreatobiliary system. Histopathology 63(5): 640-648, 2013. PMID: 24024557. DOI: 10.1111/his.12229

12 Liu SH, Lin CY, Peng SY, Jeng YM, Pan HW, Lai PL, Liu CL and Hsu HC: Down-regulation of annexin A10 in hepatocellular carcinoma is associated with vascular invasion, early recurrence, and poor prognosis in synergy with p53 mutation. Am J Pathol 160(5): 1831-1837, 2002. PMID: 12000734. DOI: 10.1016/ S0002-9440(10)61129-7

13 Kodaira H, Koma YI, Hosono M, Higashino N, Suemune K, Nishio M, Shigeoka M and Yokozaki H: ANXA10 induction by interaction with tumor-associated macrophages promotes the growth of esophageal squamous cell carcinoma. Pathol Int 69(3): 135-147, 2019. PMID: 30758105. DOI: 10.1111/pin.12771

14 Ishikawa A, Sakamoto N, Honma R, Taniyama D, Fukada K, Hattori T, Sentani K, Oue N, Yanagihara K, Tanabe K, Ohdan H and Yasui W: Annexin A10 is involved in the induction of pancreatic duodenal homeobox-1 in gastric cancer tissue, cells and organoids. Oncol Rep 43(2): 581-590, 2020. PMID: 31789399. DOI: $10.3892 /$ or.2019.7422

15 Ishikawa A, Kuraoka K, Zaitsu J, Saito A, Kuwai T, Suzuki T, Tashiro H, Taniyama $\mathrm{K}$ and Yasui W: Loss of annexin a10 expression is associated with poor prognosis in early gastric cancer. Acta Histochem Cytochem 53(5): 113-119, 2020. PMID: 33177783. DOI: $10.1267 /$ ahc.20-00014

16 Xue Y, Vanoli A, Balci S, Reid MM, Saka B, Bagci P, Memis B, Choi H, Ohike N, Tajiri T, Muraki T, Quigley B, El-Rayes BF, Shaib W, Kooby D, Sarmiento J, Maithel SK, Knight JH, Goodman M, Krasinskas AM and Adsay V: Non-ampullaryduodenal carcinomas: clinicopathologic analysis of 47 cases and comparison with ampullary and pancreatic adenocarcinomas. Mod Pathol 30(2): 255-266, 2017. PMID: 27739441. DOI: 10.1038/modpathol.2016.174

17 Gene Expression Omnibus. Available at: https://www.ncbi.nlm. nih.gov/geo/ [Last accessed on 28th January 2021]

18 Kim JK, Kim PJ, Jung KH, Noh JH, Eun JW, Bae HJ, Xie HJ, Shan JM, Ping WY, Park WS, Lee JY and Nam SW: Decreased expression of annexin A10 in gastric cancer and its overexpression in tumor cell growth suppression. Oncol Rep 24(3): 607-612, 2010. PMID: 20664964

19 Lu SH, Chen YL, Shun CT, Lai JN, Peng SY, Lai PL and Hsu $\mathrm{HC}$ : Expression and prognostic significance of gastric-specific annexin A10 in diffuse- and intestinal-type gastric carcinoma. J Gastroenterol Hepatol 26(1): 90-97, 2011. PMID: 21175800. DOI: $10.1111 /$ j.1440-1746.2010.06480.x

20 Sajanti SA, Väyrynen JP, Sirniö P, Klintrup K, Mäkelä J, Tuomisto A and Mäkinen MJ: Annexin A10 is a marker for the serrated pathway of colorectal carcinoma. Virchows Arch 466(1): 5-12, 2015. PMID: 25395067. DOI: 10.1007/s00428-014-1683-6

$21 \mathrm{Kim}$ JH, Rhee YY, Kim KJ, Cho NY, Lee HS and Kang GH: Annexin A10 expression correlates with serrated pathway features in colorectal carcinoma with microsatellite instability. APMIS 122(12): 1187-1195, 2014. PMID: 24909058. DOI: 10.1111/apm.12284

22 Tsai JH, Lin YL, Cheng YC, Chen CC, Lin LI, Tseng LH, Cheng ML, Liau JY and Jeng YM: Aberrant expression of annexin A10 is closely related to gastric phenotype in serrated pathway to colorectal carcinoma. Mod Pathol 28(2): 268-278, 2015. PMID: 25081749. DOI: $10.1038 /$ modpathol.2014.96

23 Tian J, Liu J, Guo C, Yang X, Yang Y, Gou H, Qiu M and Cao D: Prognostic factors and treatment outcomes in patients with non-ampullary small bowel adenocarcinoma: Long-term analysis. Medicine (Baltimore) 98(17): e15381, 2019. PMID: 31027129. DOI: 10.1097/MD.0000000000015381

24 Sun R, Liu Z, Qiu B, Chen T, Li Z, Zhang X, Xu Y and Zhang $\mathrm{Z}$ : Annexin10 promotes extrahepatic cholangiocarcinoma metastasis by facilitating EMT via PLA2G4A/PGE2/STAT3 pathway. EBioMedicine 47: 142-155, 2019. PMID: 31492557. DOI: 10.1016/j.ebiom.2019.08.062

25 Wang J, Zhao S, Wang F, Wang J and Zhang Y: Prognostic significance of increased expression of annexin a10 (anxa10) in serous epithelial ovarian cancer. Med Sci Monit 25: 5666-5673, 2019. PMID: 31363077. DOI: 10.12659/MSM.915911

26 Liu X, Yang M, Guo Y and Lu X: Annexin A10 is a novel prognostic biomarker of papillary thyroid cancer. Ir J Med Sci 190(1): 59-65, 2021. PMID: 32451762. DOI: 10.1007/s11845020-02263-x

27 Ushiku T, Arnason T, Fukayama M and Lauwers GY: Extraampullary duodenal adenocarcinoma. Am J Surg Pathol 38(11): 1484-1493, 2014. PMID: 25310836. DOI: 10.1097/PAS 0000000000000278 